\title{
The association between protease inhibitors and anal cancer outcomes in veterans living with HIV treated with definitive chemoradiation: a retrospective study
}

\author{
Alison K. Yoder ${ }^{1,2}$, David S. Lakomy ${ }^{2,3}$, Yongquan Dong ${ }^{4,5}$, Suchismita Raychaudhury ${ }^{4,5}$, Kathryn Royse ${ }^{4}$, \\ Christine Hartman ${ }^{4,5}$, Peter Richardson ${ }^{4,5}$, Donna L. White ${ }^{4,5}$, Jennifer R. Kramer ${ }^{4,5}$, Lilie L. Lin ${ }^{2}$ and \\ Elizabeth Chiao ${ }^{4,5^{*}}$ (D)
}

\begin{abstract}
Background: The incidence of anal squamous cell carcinoma has been increasing, particularly in people living with HIV (PLWH). There is concern that radiosensitizing drugs, such as protease inhibitors, commonly used in the management of HIV, may increase toxicities in patients undergoing chemoradiation. This study examines treatment outcomes and toxicities in PLWH managed with and without protease inhibitors who are receiving chemoradiation for anal cancer.

Methods: Patient demographic, HIV management, and cancer treatment information were extracted from multiple Veterans Affairs databases. Patients were also manually chart reviewed. Among PLWH undergoing chemoradiation for anal carcinoma, therapy outcomes and toxicities were compared between those treated with and without protease inhibitors at time of cancer treatment. Statistical analysis was performed using chi-square, Cox regression analysis, and logistic regression.

Results: A total of 219 PLWH taking anti-retroviral therapy undergoing chemoradiation for anal cancer were identified and included in the final analysis. The use of protease inhibitors was not associated with any survival outcome including colostomy-free survival, progression-free survival, or overall survival (all adjusted hazard ratio $p$-values $>0.05$ ). Regarding toxicity, protease inhibitor use was not associated with an increased odds of hospitalizations or non-hematologic toxicities; however, protease inhibitor use was associated with increased hospitalizations for hematologic toxicities, including febrile neutropenia $(p<0.01)$.

Conclusion: The use of protease inhibitors during chemoradiation for anal carcinoma was not associated with any clinical outcome or increase in non-hematologic toxicity. Their use was associated with increased hospitalizations for hematologic toxicities. Further prospective research is needed to evaluate the safety and efficacy of protease inhibitors for patients undergoing chemoradiation.
\end{abstract}

Keywords: HIV, Protease inhibitors, Anal carcinoma, Squamous cell carcinoma, Chemoradiation

\footnotetext{
*Correspondence: eychiao@mdanderson.org

${ }^{4}$ Department of Medicine, Baylor College of Medicine, 1155 Pressler St. Unit, Houston 1340, USA

${ }^{5}$ Michael E. DeBakey Veterans Affairs Medical Center, Houston, TX, USA

Full list of author information is available at the end of the article
}

(c) The Author(s). 2021 Open Access This article is licensed under a Creative Commons Attribution 4.0 International License, which permits use, sharing, adaptation, distribution and reproduction in any medium or format, as long as you give appropriate credit to the original author(s) and the source, provide a link to the Creative Commons licence, and indicate if changes were made. The images or other third party material in this article are included in the article's Creative Commons licence, unless indicated otherwise in a credit line to the material. If material is not included in the article's Creative Commons licence and your intended use is not permitted by statutory regulation or exceeds the permitted use, you will need to obtain permission directly from the copyright holder. To view a copy of this licence, visit http://creativecommons.org/licenses/by/4.0/. The Creative Commons Public Domain Dedication waiver (http://creativecommons.org/publicdomain/zero/1.0/) applies to the data made available in this article, unless otherwise stated in a credit line to the data. 


\section{Background}

Cancers of the anus constitute approximately 8300 new cases and 1280 deaths per year in the United States [1]. While anal cancer remains a relatively rare malignancy, its incidence has steadily risen [2]. In particular, the introduction of highly active antiretroviral therapy (ART) and the subsequent shift of HIV into a chronic condition has played an important role in this increase [2]. As several studies have shown, HIV-infection with ART therapy is associated with both an increase in the risk of anal cancer as well as higher relapse rates following treatment [3-5].

Chemoradiation (CRT) is the mainstay of treatment for anal cancer that has any nodal involvement or is greater than T1 (and select T2) at diagnosis [6]. Radiation typically consists of a dose of 50-54 Gy to gross disease [7]. While several chemotherapy regimens can be utilized, the most widely used consists of 5-flurouracil (5-FU) and mitomycin C [8], however, 5-flurouracil and cisplatin [9] or capecitabine with mitomycin [10] are acceptable alternatives per National Comprehensive Cancer Network (NCCN) guidelines [11]. Regardless of regimen used, however, toxicity concerns abound. The most common toxicities consist of hematologic, dermatologic, gastrointestinal, and genitourinary complications [7-10]. These toxicities are often so severe that therapy completion rates remains a challenge as many trials have shown significant gaps, delays, or inability to complete full chemotherapy regimens [12].

Thus, there are concerns that drugs that serve as radiosensitizers might be difficult to tolerate during radiation treatment, possibly rendering them contraindicated. One auspicious class of radiosensitizing agents includes protease inhibitors (PIs) targeted against HIV-1. PIs have demonstrated in vitro and in vivo radiosensitizing effects in a variety of tumor cell lines [13-16]. Due to the promising preclinical data, several phase I studies testing the HIV-1 protease inhibitor nelfinavir combined with chemoradiation have been conducted for multiple cancer sites with promising results [17-24]. In one study of concurrent nelfinavir with CRT for non-small cell lung cancer [18], for example, they saw a median survival of 41.1 months, which was beyond historic norms for standard CRT of 17.0 months [25]. While treatment outcomes for PI results are promising, the question remains as to whether the radiosensitization from PIs, which lends to its increased efficacy, may also result in enhanced toxicity of normal tissue. Although randomized clinical trials would be the optimal avenue through which to study PI radiosensitization, people living with HIV (PLWH) are a natural cohort of patients who regularly take these medications during treatment for anal cancer. In this study we retrospectively analyze data from the Department of Veterans Affairs to examine the effect of PI usage during CRT for anal squamous cell carcinoma on survival and toxicities.

\section{Methods \\ Patient selection and characteristics}

Patient information and data was extracted from the United States Department of Veterans Affairs (VA) databases and registries accessible through the VA Informatics and computing infrastructure (VINCI), including the VA Corporate Data Warehouse (CDW) and the VA Central Cancer Registry (CCR) from years 2000 to 2016. The CDW is an automated VA database that includes all patient data including inpatient and outpatient encounters, laboratory information, and pharmacy information. It is updated in near real-time and is available for all VA users. The VA CCR is a national data repository for over 750,000 VA patients with cancer $[26,27]$. Each VA medical center has its own cancer registrar who manually extracts the data, which is then aggregated into a national cancer registry. Data was abstracted from both databases. VINCI was then used to manually chart review the data pulled from CDW and CCR as well as to extract additional variables not available in the databases.

Inclusion criteria for patients included veterans with HIV-positivity, biopsy-proven anal squamous cell carcinoma, at least 18 years of age at start of treatment, definitive CRT treatment, and on ART during treatment. HIV-infection was determined as "positive" as described by Kramer et al. [28], whereby patients must fulfill two of the three following criteria: (1) at least one positive HIV antibody test by ELISA or Western Blot, viral load (any \pm interderminate), or tested for CD4+ count; (2) at least one inpatient or outpatient pharmacy records for HIV ART medication; and (3) International Classification of Diseases, Ninth Revision (ICD-9) (042 or V08) or ICD-10 code (B20 or Z21) for HIV. Anal cancer was identified via primary site and histology codes 15401548, 2304-2306, C19-20, C210-212, D011-013 as well as text searches; biopsy-proven confirmation was determined through chart review. The type of ART medication classified as PI vs. non-PI based was based on the medication the patient was taking at the start of chemoradiation. Exclusion criteria included patients who were diagnosed with HIV after CRT for anal cancer, patients treated with palliative intent, and those who were treated with primary surgery (see Fig. 1).

Patient information collected included patient characteristics such as age at diagnosis, race/ethnicity, body mass index (BMI), smoking and alcohol use, history of prior cancers; disease stage at diagnosis; HIV-related variables including any changes in type of ART used within 90 days of starting treatment, CD4 at diagnosis and nadir CD4 during radiation; treatment variables including radiation dose and modality, duration, and 


\section{HIV+ Patients}

$18+$ years

ICD-10 code for anal cancer OR

text search for anal carcinoma

\section{Patients}

Biopsy-proven anal squamous cell carcinoma

Excluded: Diagnosed with HIV after

treatment for anal carcinoma

\section{Patients}

Treated with definitive-intent chemoradiation

Received anti-retroviral therapy during treatment

\section{Patients}

Fig. 1 Patient Selection

completion, and chemotherapy regimen including if there was dose reduction; outcome data including recurrence date and site, receipt of colostomy and date, and vital status as of May 2019; and toxicity information including acute and chronic toxicities as well as risk of hospitalization. Toxicities were based on the National Cancer Institute's Common Terminology Criteria for Adverse Events Version 5.0.

\section{Statistical analysis}

We performed chi-square and Fisher's exact analysis to compare sociodemographic risk factors, clinical characteristics and outcomes among PLWH who received PIs during treatment to those who did not (i.e., non-PI users). Kaplan-Meier curves were constructed, and logrank tests were performed to compare survival curves for PI users and non-PI users for overall survival (OS), recurrence-free survival (RFS) and colostomy-free survival (CFS). Cox proportional hazards (PH) analysis was performed to assess for differences in RFS, CFS, and OS, respectively, in PI users and non-users. We interrogated all variables that on univariate analysis had an overall $p$ - value or individual stratum specific Wald test $p$-value for non-binary variables of $<0.25$ to allow for potential heterogeneity as well as race/ethnicity and age regardless of significance in multivariable analyses. We employed backward stepwise selection to final reported multivariable models for OS, RFS, and CFS in PI users and nonusers with other covariates retained in final reported model if overall or stratum-specific $p$-value $<0.10$, and with race/ethnicity and age forced in final model regardless of significance given their known strong association with cancer risk and to aid comparison with other studies. We employed this approach in multivariable analyses to maximize precision of effect estimates for our primary variable of interest, PI usage vs. non-usage, and to minimize risk of overfitting while also adjusting for race/ ethnicity and age given they are well-established risk factors for most cancers and any other strongly associated covariates. Effect estimates are reported as hazard ratios (HR) with associated 95\% confidence intervals (CIs) and $p$-values, with two-sided $p$-values less than $<0.05$ considered significant and $p$-values between 0.05 and 0.10 considered approaching significance. 
To analyze toxicity, logistic regression analysis was performed to analyze variables associated with $\geq$ grade 3 acute and late toxicities as well as hospitalizations. Acute toxicities were defined as occurring within 90 days of starting treatment; late toxicities were defined as occurring at least 90 days after starting treatment until the end of follow-up. Hospitalizations were included in analysis if they occurred during treatment or within 90 days of treatment onset. Our logistic regression modeling approach was the same as described for Cox models above including use of the same criteria in univariable analyses followed by multivariable analysis. We also employed logistic regression to examine the association between the use of PIs and relative risk of specific treatment related outcomes as well as of all specific individual toxicities. However, given the even further limited sample size for analyses evaluating specific individual toxicities, our primary analyses for them is our univariable analysis. For purposes of hypothesis generation we also performed exploratory multivariable analyses for specific treatment and toxicity related outcomes significant at $p<0.05$ given further reduction in study power. Logistic regression-based model results are presented as odds ratios with associated $95 \%$ confidence intervals and $p$ values, with two-sided $p$-values $<0.05$ considered statistically significant. SAS v9.4 was used for analysis.

\section{Results}

\section{Patient and HIV characteristics}

A total of 339 veteran patients were found to be HIVpositive and diagnosed with anal squamous cell carcinoma. Of these, 219 PLWH were treated with definitiveintent CRT and received at least one anti-retroviral drug during their treatment and were included in the final analysis (Table 1). Median age at time of HIV diagnosis was 46 years (interquartile range (IQR) 40-53) and median age at time of anal cancer diagnosis was 53.7 years (IQR 48.9-60.8). Median follow up was 4.7 years (IQR 2.1-8.6). The majority of patients were Caucasian $(50.7 \%, n=111)$, and nearly half were stage II at diagnosis $(48.9 \%, n=107)$. Most patients had smoked cigarettes at some point during their lifetime $(68.9 \%, n=151)$, and $51.6 \%(n=113)$ still smoked at the time of cancer diagnosis. There was no significant difference in age, race/ ethnicity, alcohol use, or smoking status between PLWH who were treated with PIs and those who were not ( $p=$ $0.19,0.78,0.73$, and 0.80 respectively). PLWH treated with PIs were significantly less likely to be obese; only $9.2 \%$ had a BMI at time of treatment of 30 or greater, compared to $16.7 \%$ of PLWH who were not treated with PIs $(p=0.05)$.

Most patients had CD4 count greater than 200 (cells/ $\mathrm{mL})$ at time of cancer diagnosis $(72.6 \%, n=159)$. The majority of patients were on PIs at time of treatment,
(69.9\%, $n=153)$. PLWH treated with PIs were less likely to have CD4 count greater than 200 at time of HIV diagnosis $(38.6 \%, n=59$ vs $63.6 \%, n=42 ; p<0.01)$ or at time of cancer diagnosis $(66.0 \%, n=101$ vs $87.9 \%, n=58$; $\mathrm{p}<$ 0.01). Twenty-five patients changed ART medications in the 90 days following the start of radiation treatment. An additional sensitivity analysis was done limiting the cohort to only the patients who did not change the type of medication they were taking, which showed no significant difference between the two cohorts in either univariate or multivariable analysis (data not shown).

\section{Chemoradiation treatment characteristics and outcome analysis}

All patients included in this study were treated with curative-intent CRT, though 25 patients (11.4\%) did not complete all fractions of radiation. There was no significant difference in radiation completion between those who received PIs and those who did not $(p=0.48)$. Seventy-five PLWH (34.2\%) received intensity-modulated radiation therapy (IMRT), $75(34.2 \%)$ received $2 \mathrm{D}$ or $3 \mathrm{D}$ radiation, and $69(31.5 \%)$ did not have this information available. The median radiation dose was 54 Gy. 5-FU and mitomycin was the most common chemotherapy regimen with 162 patients (74.0\%) receiving this treatment. Patients who were on PIs were more likely to receive 5-FU and cisplatin than patients who were not taking PIs $(13.1 \%, n=$ 20 vs $6.1 \%, n=4 ; p=0.05)$. A significant percentage of patients required a chemotherapy dose reduction $(29.7 \%$, $n=65)$ during their treatment.

There was no significant difference in any outcome measure between PLWH who took PIs and those that did not (Fig. 2). On univariate Cox analysis, there were no significant differences with OS, RFS, or CFS in non-users of PIs when compared to patients who did receive PIs (HR 1.04 [0.68-1.59], 1.53 [0.86-2.72], 1.07 [0.52-2.23], respectively; all $p>0.05$ ) (Table 2). Increasing stage was associated with both worse OS as well as increased chance of tumor recurrence (OS HR 2.38 [1.17-4.82], 2.94 [1.167.41], 3.07 [1.44-6.53], and 3.83 [1.18-12.47] for stage II, IIIA, IIIB, and IV, respectively; all $p<0.05)$. Race/ethnicity, age, and alcohol use were not associated with OS, RFS, or CFS (all $p>0.5$ ).

In the multivariable analysis, the use of PIs continued to have no significant association with OS, RFS, or CFS (HRadjusted (adj) 0.86 [0.53-1.38], 1.34 [0.74-2.43], 1.03 [0.49-2.15], respectively; all $p>0.05)$. Increasing stage remained significantly associated with both OS and RFS; however, stage was not associated with CFS and therefore it was not retained in the final model. Neither race nor age were significantly associated with OS, RFS, or CFS. PLWH with a BMI between 25 and 29.9, or clinically overweight, had improved OS than those who with BMI $<25$ or normal weight (HRadj 0.49 [0.30-0.81], $p<$ 
Table 1 Demographic and treatment variables during chemoradiation

Taking protease inhibitors

n (\%)

$153(69.86)$

\section{Age at diagnosis}

$<40$

$40-59$

$60+$

Race/Ethnicity

African American
Caucasian
Hispanic
Other/Unknown

Year HIV Diagnosed

$\begin{array}{ll}<2000 & 103(67.32) \\ 2000-2009 & 39(25.49) \\ 2010-2016 & 11(7.19)\end{array}$

\section{Smoker}

$\begin{array}{ll}\text { Current } & 76(49.67) \\ \text { Former } & 28(18.30) \\ \text { Never } & 41(26.80) \\ \text { Unknown } & 8(5.23)\end{array}$

\section{Stage}

$\|$

IIIA

IIIB

IV

Unknown

CD4 at HIV Diagnosis

$$
\begin{aligned}
& \leq 200 \\
& >200 \\
& \text { Unknown }
\end{aligned}
$$

$21(13.73)$

$73(47.71)$

$15(9.80)$

35 (22.88)

6 (3.92)

$3(1.96)$

55 (35.95)

59 (38.56)

$39(25.49)$

\section{CD4 at Cancer Diagnosis}

$$
\leq 200
$$$$
>200
$$

Unknown

Nadir CD4 during Radiation

$$
\begin{aligned}
& \leq 200 \\
& >200
\end{aligned}
$$$$
\text { missing }
$$

BMI

$$
\begin{aligned}
& <25 \\
& 25-29.9 \\
& \geq 30 \\
& \text { Unknown }
\end{aligned}
$$

43 (28.10)

101 (66.01)

9 (5.88)

79 (51.63)

$41(26.80)$

76 (49.67)
53 (34.64)

14 (9.15)

10 (6.54)
Not taking protease inhibitors

n (\%)

$66(30.14)$

$2(3.03)$
$40(60.61)$
$24(36.36)$

$21(31.82)$

33 (50.00)

7 (10.61)

$5(7.58)$

39 (59.09)

$23(34.85)$

4 (6.06)

0.80

$37(56.06)$

10 (15.15)

15 (22.73)

$4(6.06)$

0.28

$15(22.73)$

$34(51.52)$

$3(4.55)$

13 (19.70)

1 (1.52)

$0(0)$

$<0.01$

20 (30.30)

42 (63.64)

4 (6.06)

8 (12.12)

58 (87.88)

$0(0)$

$20(30.30)$

25 (37.88)

21 (31.82)

0.05

0.78

0.19

\section{(9)}


Table 1 Demographic and treatment variables during chemoradiation (Continued)

\begin{tabular}{|c|c|c|c|}
\hline & $\begin{array}{l}\text { Taking protease inhibitors } \\
\text { n (\%) }\end{array}$ & $\begin{array}{l}\text { Not taking protease inhibitors } \\
\text { n (\%) }\end{array}$ & $p$-value \\
\hline & $153(69.86)$ & $66(30.14)$ & \\
\hline Alcohol Use & & & 0.73 \\
\hline Yes & $57(37.25)$ & $23(34.85)$ & \\
\hline No & $96(62.75)$ & $43(65.15)$ & \\
\hline History of prior cancer & & & 0.14 \\
\hline Yes & $22(14.38)$ & $14(21.21)$ & \\
\hline No & $129(84.31)$ & $49(74.24)$ & \\
\hline Unknown & $2(1.31)$ & $3(4.55)$ & \\
\hline Radiation Modality & & & 0.87 \\
\hline $2 D+3 D$ & $54(35.29)$ & $21(31.82)$ & \\
\hline IMRT & $52(33.99)$ & $23(34.85)$ & \\
\hline Unknown & $47(30.72)$ & $22(33.33)$ & \\
\hline Completed Radiation Treatment & & & 0.48 \\
\hline Yes & $134(87.58)$ & $60(90.91)$ & \\
\hline No & $19(12.42)$ & $6(9.09)$ & \\
\hline Length of Radiation Treatment & & & 0.59 \\
\hline$\leq 50$ days & $86(56.21)$ & $42(63.64)$ & \\
\hline$>50$ days & $61(39.87)$ & $22(33.33)$ & \\
\hline Unknown & $6(3.92)$ & $2(3.03)$ & \\
\hline Chemotherapy Regimen & & & 0.05 \\
\hline $5 \mathrm{FU}+$ mitomycin $\mathrm{C}$ & $115(75.16)$ & $47(71.21)$ & \\
\hline $5 F U+$ cisplatin & $20(13.07)$ & $4(6.06)$ & \\
\hline other & $18(11.76)$ & $15(22.73)$ & \\
\hline Chemotherapy Dose Reduction & & & 0.25 \\
\hline Yes & $49(32.03)$ & $16(24.24)$ & \\
\hline No & $104(67.97)$ & $50(75.76)$ & \\
\hline
\end{tabular}

0.01). In addition, those treated with IMRT had improved OS than those treated with either $2 \mathrm{D}$ or $3 \mathrm{D}$ radiation (HRadj 0.50 [0.29-0.86], $p=0.01$ ).

Patients who completed radiation treatment had significantly improved OS and RFS than those that did not in multivariable analysis (both $p<0.05$ ). There was no association between PLWH who took PIs and their ability to complete radiation treatment $(p=0.48)$, or the length of radiation treatment $(p=0.33)$ (Table 4). PLWH who required a dose reduction of chemotherapy had decreased OS and RFS, though these were not statistically significant findings (HR 1.40 [0.94-2.08], 1.46 [0.902.39 ]; respectively) (Table 2). There was no association between the use of PIs and patients requiring dose reductions in chemotherapy $(p=0.25)$ (Table 4$)$.

\section{Toxicity analysis}

The use of PIs did not increase the risk for either acute or long-term non-hematologic toxicity in PLWH treated with definitive CRT for anal cancer in either univariate or multivariable logistic regression analysis (all $p>0.05$, Table 3). However, patients on PI ART regimens had an increased risk of hospitalization (Odds ratio (OR) 2.44 [1.27-4.65], $p<0.01)$ compared to those who did not take PIs on univariate analysis. This association continued after controlling for confounding variables in the multivariable analysis (ORadj 2.17 [1.04-4.56], $p=0.04$ ).

The majority of patients experienced grade 3 or higher hematologic toxicity $(62.6 \%, n=137)$. Patients who were taking PIs were significantly more likely to have any hematologic toxicity greater than grade 3 in univariable analysis (OR 2.12 [1.18-3.83], $p=0.01$, Table 4). Specifically, they were more likely to experience at least grade 3 lymphopenia during treatment (OR 1.92 [1.05-3.51], $p=0.03)$. While grade 3 or greater leukopenia was more likely in patients on PI ART (OR 1.73 [0.93-3.23], $p=$ $0.08)$, this was not a statistically significant finding. 


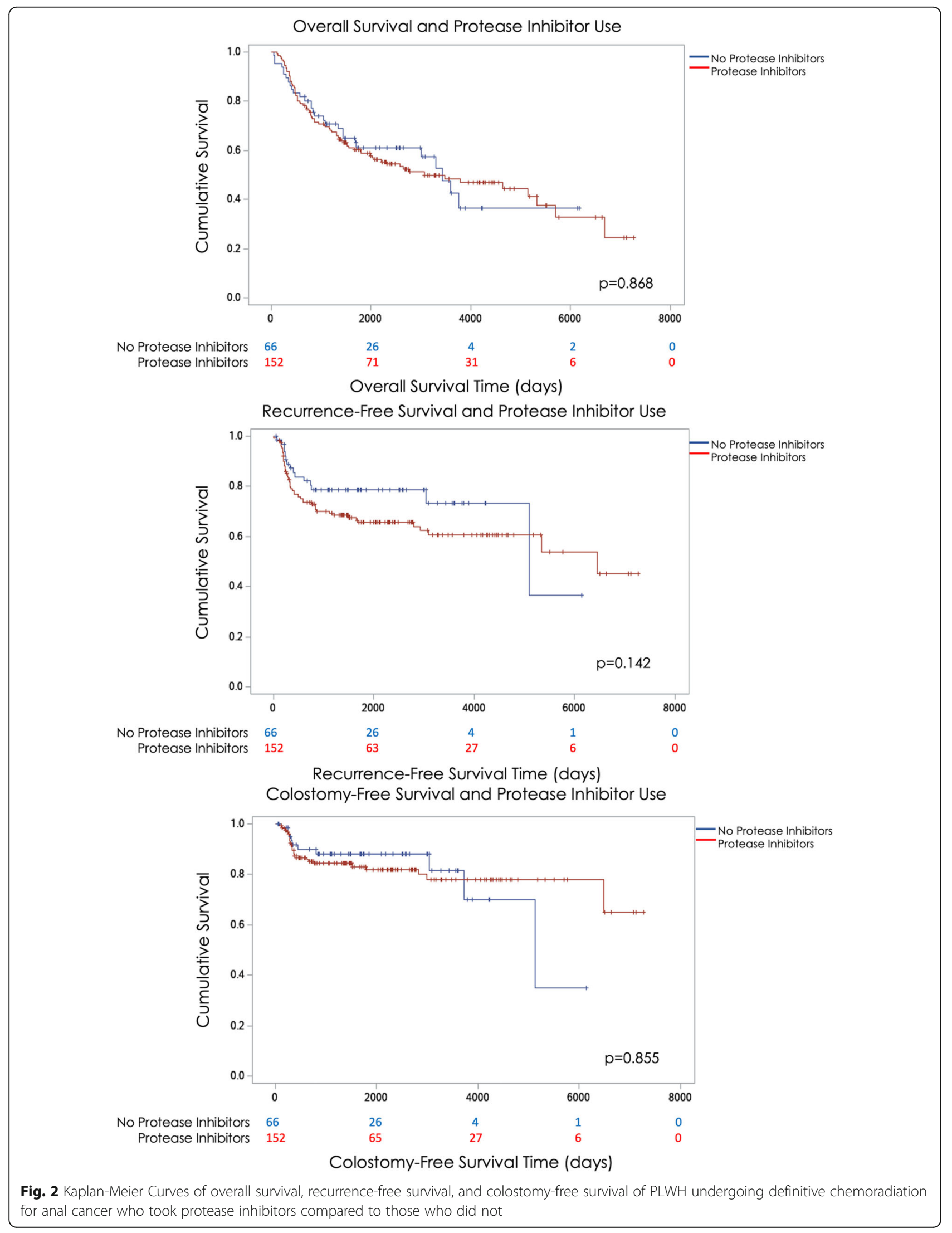




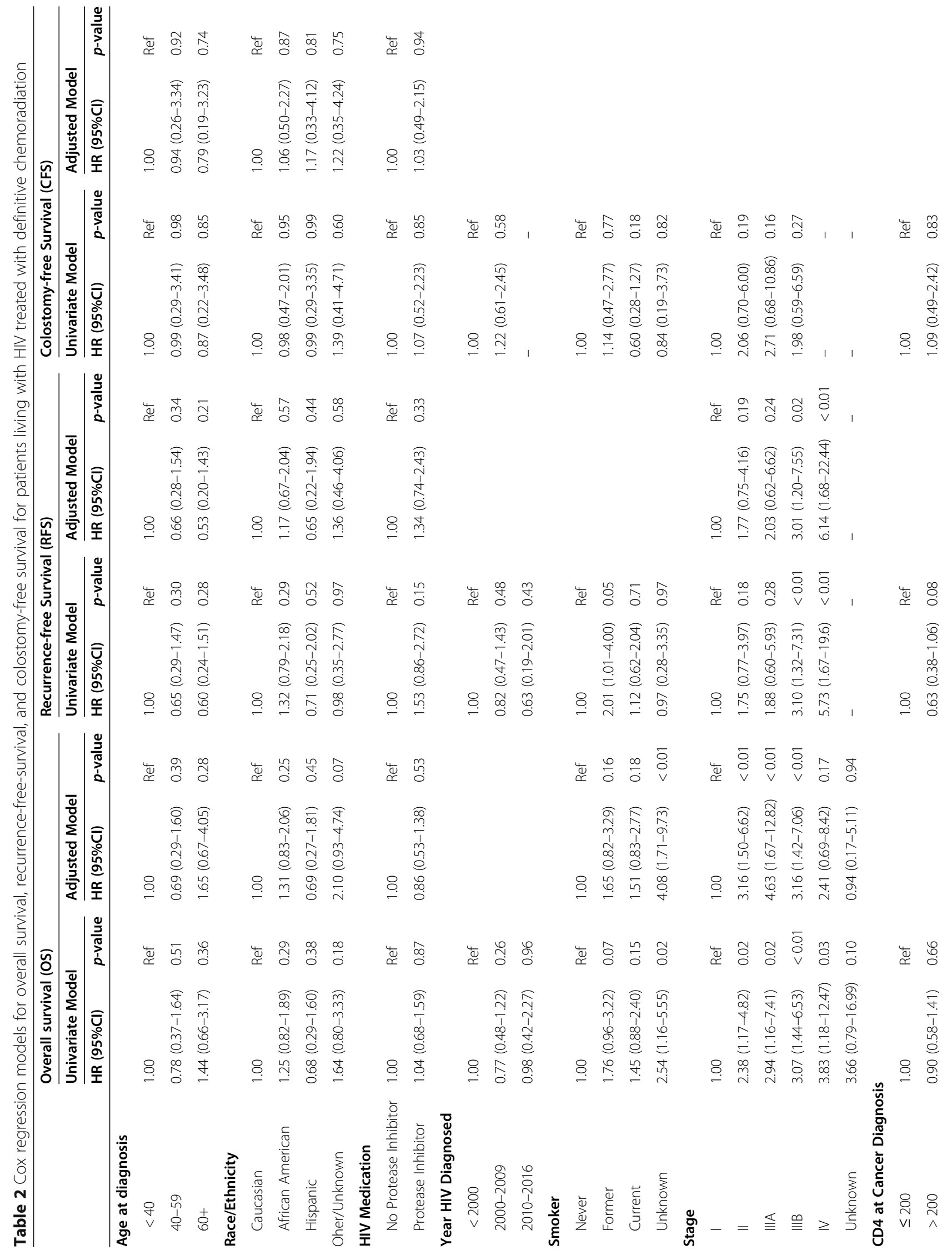




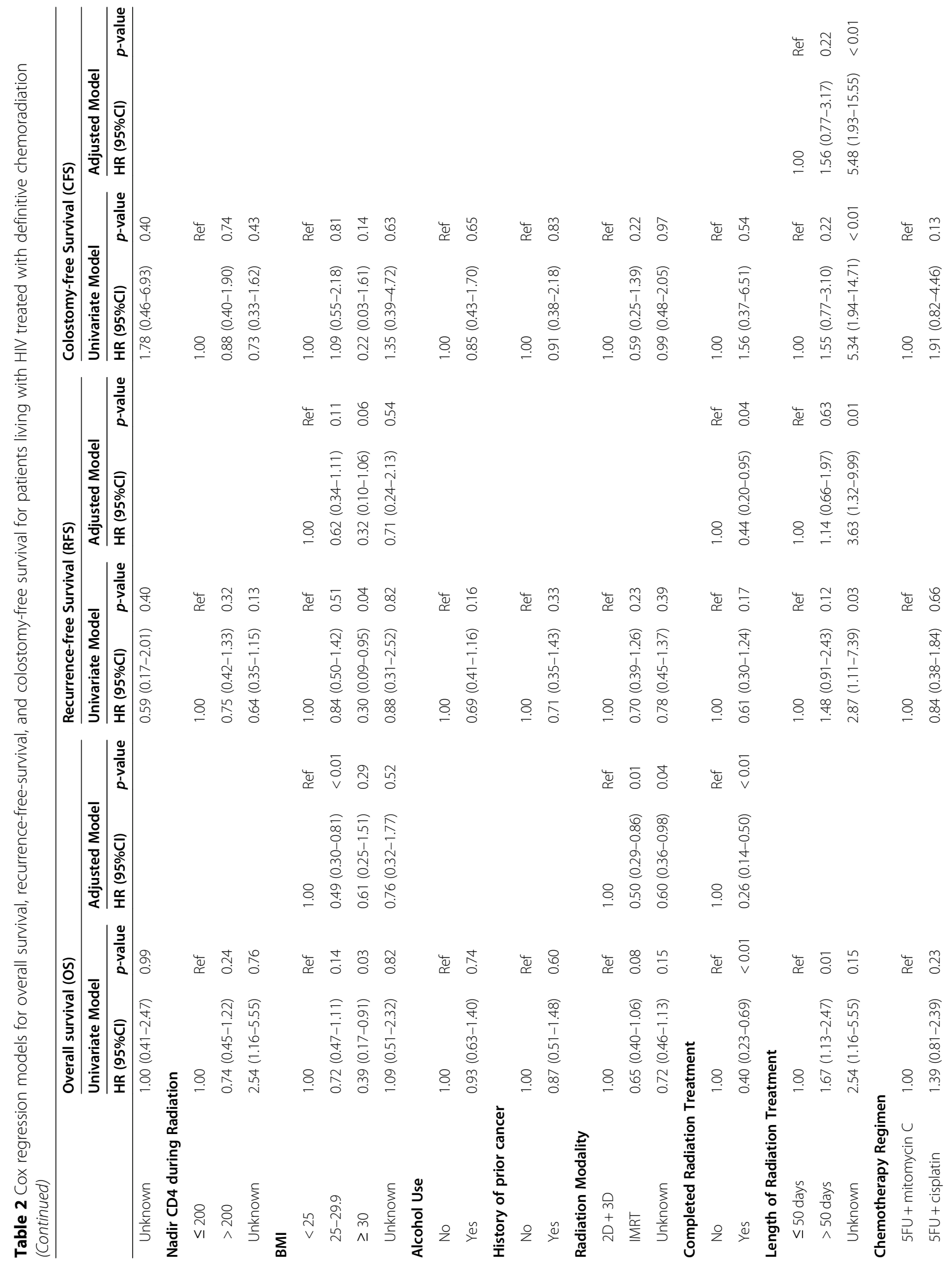




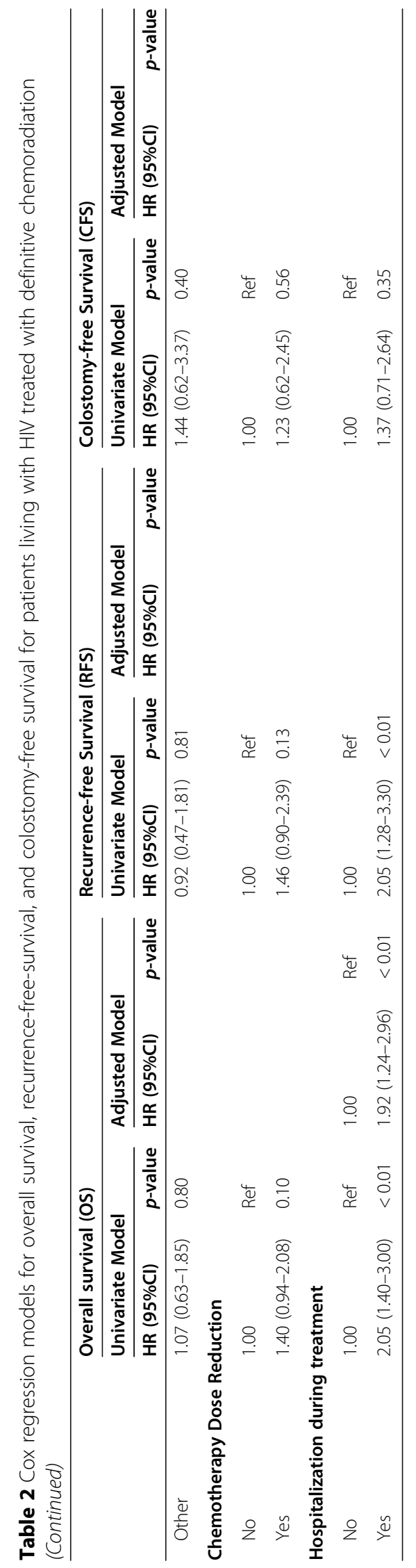




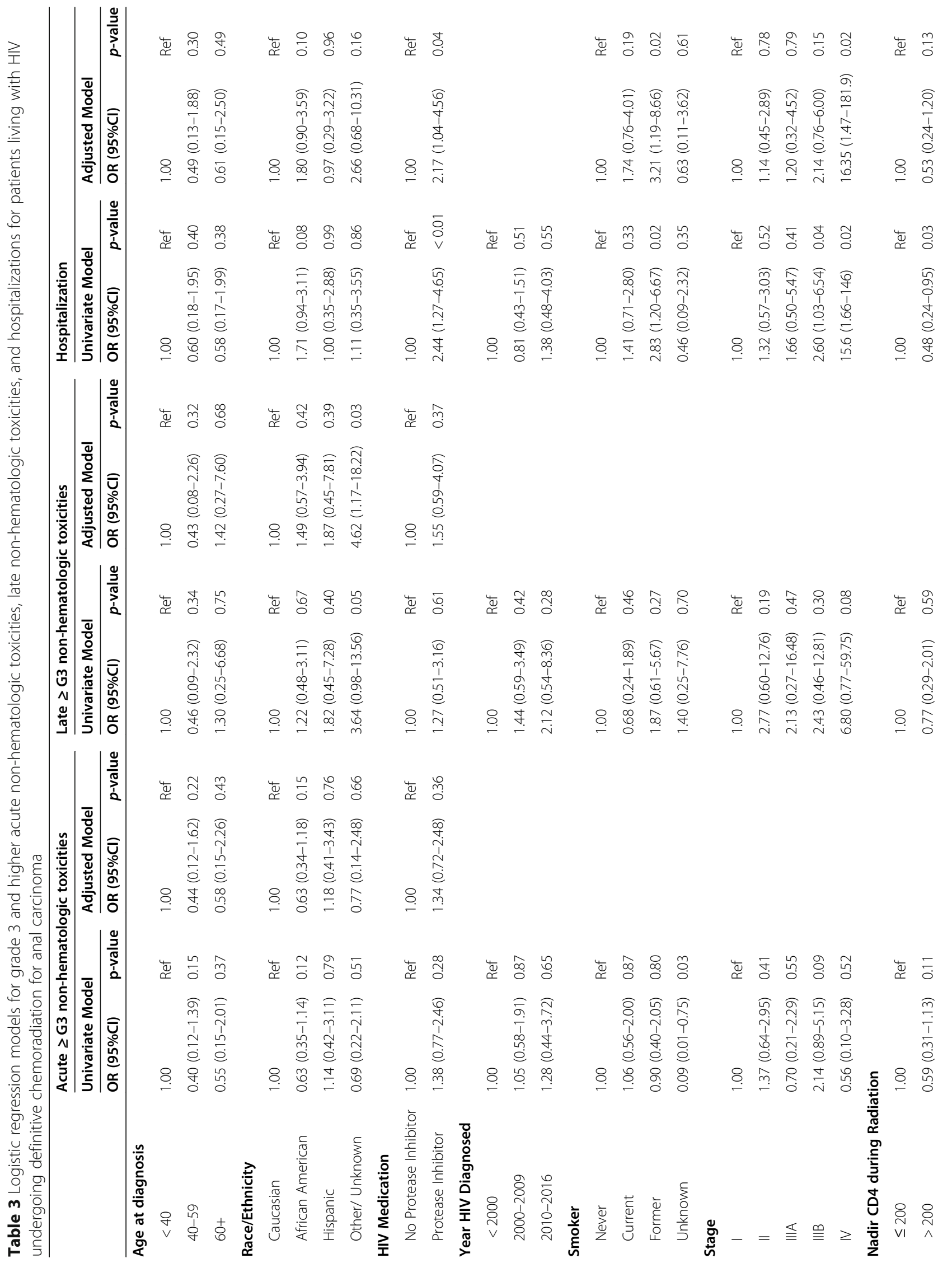




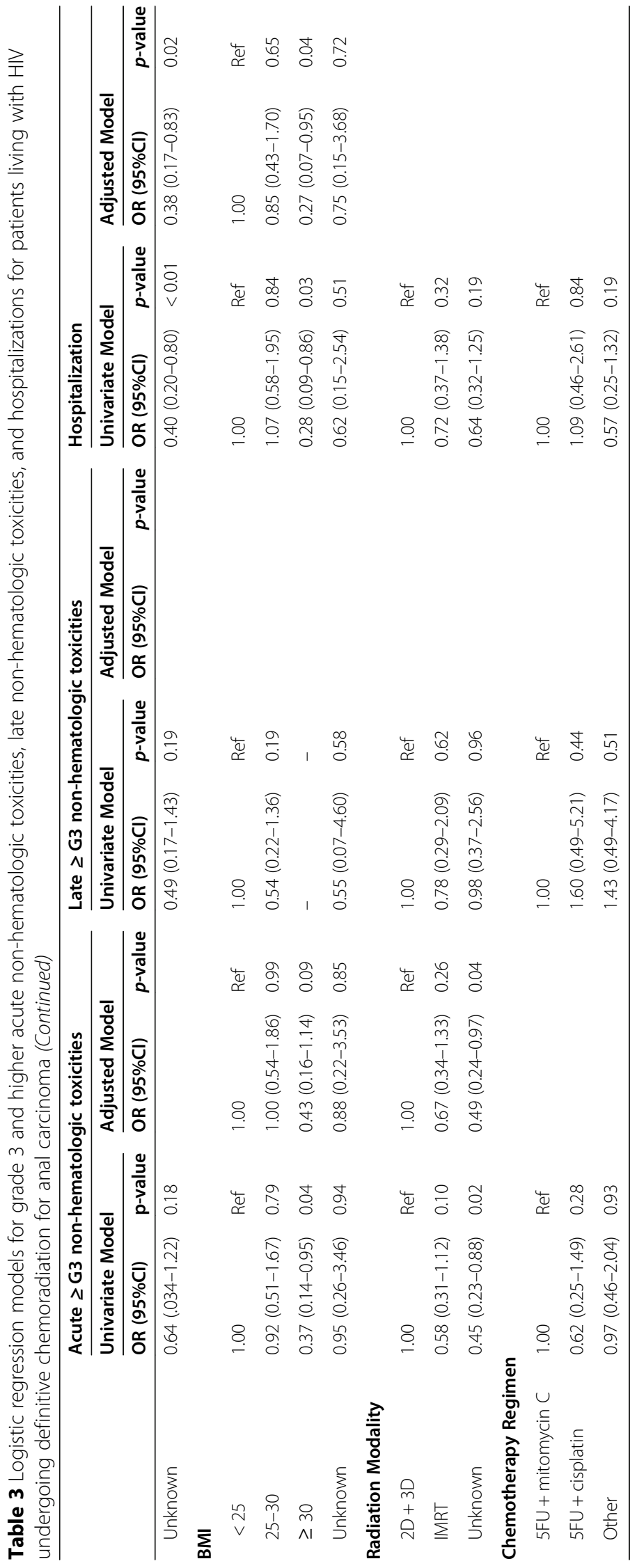


Table 4 Univariate Logistic Regression models for relative risk of select individual treatment and toxicity related outcomes according to PI use

\begin{tabular}{|c|c|c|}
\hline Outcome & $\begin{array}{l}\text { Taking a Protease Inhibitor } \\
\text { Odds Ratio }(95 \% \mathrm{CI})\end{array}$ & $p$-value \\
\hline \multicolumn{3}{|l|}{ Treatment-related } \\
\hline Completed Radiation & $0.71(0.27-1.86)$ & 0.48 \\
\hline Length of Radiation Treatment $>50$ days & $0.74(0.40-1.36)$ & 0.33 \\
\hline Required a Chemotherapy Dose Reduction & $1.47(0.76-2.84)$ & 0.25 \\
\hline \multicolumn{3}{|l|}{ Toxicities during treatment } \\
\hline Hospitalized for GI Toxicity & $1.01(0.25-4.02)$ & 0.99 \\
\hline Hospitalized for Hematologic Toxicity & $4.60(1.56-13.5)$ & $<0.01$ \\
\hline Hospitalized for Febrile Neutropenia & $6.25(1.44-27.2)$ & 0.01 \\
\hline Hospitalized for Radiation Dermatitis & $0.74(0.21-2.63)$ & 0.65 \\
\hline Acute Radiation Dermatitis at least Grade 3 & $0.94(0.51-1.74)$ & 0.85 \\
\hline Acute GI Toxicity at least Grade 3 & $1.94(0.70-5.39)$ & 0.20 \\
\hline Acute Oral Mucositis at least Grade 3 & $1.04(0.35-3.07)$ & 0.95 \\
\hline Late GI Toxicity at least Grade 3 & $1.56(0.49-4.93)$ & 0.45 \\
\hline Other Late Toxicity at least Grade 3 & $0.63(0.17-2.32)$ & 0.49 \\
\hline Any Acute Hematologic Toxicity & $2.12(1.18-3.83)$ & 0.01 \\
\hline Acute Anemia at least Grade 3 & $1.47(0.39-5.52)$ & 0.57 \\
\hline Acute Leukopenia at least Grade 3 & $1.73(0.93-3.23)$ & 0.08 \\
\hline Acute Thrombocytopenia at least Grade 3 & $1.54(0.71-3.34)$ & 0.28 \\
\hline Acute Neutropenia at least Grade 3 & $1.73(0.71-4.20)$ & 0.23 \\
\hline Acute Lymphopenia at least Grade 3 & $1.92(1.05-3.51)$ & 0.03 \\
\hline
\end{tabular}

There was no difference in neutropenia, anemia, or thrombocytopenia in univariable analyses (all $p>0.05$ ).

PLWH on PI regimens were more likely to be hospitalized for febrile neutropenia or other hematologic toxicity than those who did not receive PIs (OR 6.25 [1.4427.2], $p=0.01$; OR 4.60 [1.56-13.5], $p<0.01$, respectively). Only $3.5 \%$ of patients $(n=3)$ who were not on PIs at time of CRT were hospitalized for febrile neutropenia, and $7 \%(n=6)$ were hospitalized for another hematologic toxicity. This is compared to $25(16.3 \%)$ patients receiving PIs that were hospitalized for febrile neutropenia, and $35(22.9 \%)$ that were hospitalized for any other hematologic toxicity. Hospitalizations during treatment was associated with decreased OS (HRadj 1.92 [1.242.96], $p<0.01$ ) on multivariable analysis (Table 2). In exploratory multivariable models for the four treatment related toxicities significantly associated with PI use in univariable analysis, all remained similarly significantly associated in multivariable analysis (e.g., ORadj 5.25 95\% CI $(1.67-16.5) \mathrm{p}<0.01$ for PI users compared to non-PI users for hospitalized for hematologic toxicity, data not shown).

There were no grade 3 or greater genitourinary complications seen in PLWH who did not receive PIs, and only two amongst those who did, a non-statistically significant difference. The use of PIs was not associated with any other specific acute or late non-hematologic toxicity on univariate analysis, including dermatitis, gastrointestinal complications, or oral mucositis (all $p>$ 0.05) (Table 4).

\section{Discussion}

In this retrospective study, we assessed survival outcomes and toxicity in anal cancer patients treated with definitive CRT in PLWH managed both with and without PI use. PI use was not significantly associated with any change in survival, recurrence, or need for a colostomy. In regard to toxicity, while PI use was associated with an increase in hospitalizations attributable to hematologic toxicities, including febrile neutropenia, no such association was found for other common toxicities including dermatologic or gastrointestinal complications. In addition, PI use was not associated with any chemotherapy or radiation treatment variable that was found to be associated with survival outcomes, such as the ability to complete radiation treatment.

Protease inhibitors, originally developed for HIVtreatment, have long shown anti-cancer effects. The etiology underlying this additional characterization is pleiotropic in nature. For one, PIs have shown direct cytotoxic effects including increased cell apoptosis due to increased endoplasmic reticulum stress and 
autophagy [29]. Additionally, for virally associated cancers, such as Human Papilloma Virus-induced anal and cervical cancer, PIs have been shown to target viral antigens that are necessary for viral replication, i.e. they have been observed to inhibit viral E6-mediated degradation of p53 [30]. Lastly, the most prominently researched anti-tumor mechanism is thought to be its downregulation of mitogenic growth signaling pathways, specifically PI3K/Akt [13, 14, 16, 31, 32]. Importantly, it is this final mechanism that is thought to contribute to PIs radiosensitizing effects. Inhibition of the PI3K/Akt pathway reduces vascular endothelial growth factor and hypoxiainducible factor $1 \alpha$ which in turn leads to increased oxygenation and enhanced radiosensitivity [16]. This effect has been demonstrated in pre-clinical bladder [13], lung [13], and head and neck tumor xenografts [16] as well as clinical studies at a variety of sites including phase $1 / 2$ non-small cell lung cancer [17, 18], phase I glioblastoma-multiforme [19], phase I cervical cancer $[20,21]$, phase I and II pancreatic cancer [22, 23], and phase I rectal cancer [24], which collectively have shown promising activity. Specifically regarding the phase I rectal cancer study, which represents an adjacent cancer site, concurrent use of the protease inhibitor nelfinavir with hypofractionated radiation therapy resulted in 5/9 patients achieving regression as well as increased blood perfusion as assessed by perfusion-CT [24].

Thus, while protease inhibitors have shown promising findings, fears that enhanced radiosensitization may result in increased toxicity and associated treatment complications persist. While this literature remains relatively novel, toxicity for combined radiotherapy-PI use has been found to be tolerable. One retrospective study of PLWH receiving radiotherapy for a variety of cancer sites found no difference in toxicity rates with or without PI use [33]. Additionally, the phase I-II studies discussed above cumulatively show overall tolerability of chemoradiotherapy with nelfinavir at the recommended FDA dose as an anti-retroviral agent in patients who are HIV negative in multiple tumor sites.

Although our study found increased hospitalizations due to acute hematologic toxicities in PLWH treated with PIs during chemoradiation, no other acute or chronic toxicity difference was found. It is important to take these results in the context of several factors. For one, it is necessary to note that the patients using PIs in our report were at greater baseline hematologic risk, as evidenced by the significantly greater proportion of patients with a CD4 count equal to or less than 200 at time of cancer diagnosis $(28.1 \%$ vs $12.1 \%, p<0.01)$. Secondly, PIs have also been found to potentiate myelotoxicity in some chemotherapy regimens due to alterations in the metabolism of chemotherapy agents via CYP3A4 enzyme inhibition [34]. And finally, the PI cohort had a higher degree of patients treated with the historically hemotoxic chemotherapy regimens that utilized mitomycin, 5-FU, and cisplatin, compared to the non-PI group. Thus, while this enhanced toxicity may be secondary to PI-based radiosensitization, there remains a multitude of factors to consider including potential drug interactions. Overall, it is vital to approach these results with the context that anal cancer treatment toxicity is often severe enough to prolong treatment duration and limit treatment completion, both of which have been observed to worsen overall outcomes [35-37]. In our study we found that PI use was not associated with prolonged duration of treatment, dose reduction of chemotherapy, or any change in radiation completion rate.

While the use of PIs was associated with an increased risk of hospitalization due to hematologic toxicity, and hospitalizations were associated with decreased OS, PI use itself was not associated with OS on either univariate or multivariable analysis. This may be due to the fact that while PIs increase hospitalizations due to neutropenia or lymphopenia, hospitalization may be attributable to a multitude of other factors that play a more significant role in OS. For example, hospitalizations due to hematologic toxicities are often easily treated (via granulocyte colony stimulating factors, or appropriate antibiotics for infections, etc.) than hospitalizations due to other treatment toxicities, such as radiation dermatitis, which may more substantially prolong or disrupt treatment. In addition, it may also be a cumulative effect of multiple toxicities that is needed to elicit those worsened outcomes rather than hematologic toxicities alone.

Another interesting finding was that patients with BMI between 25 and 30, or clinically overweight though non-obese, had improved OS compared to those with BMI $<25$ or with normal BMI in multivariable Cox regression analysis. Likely, BMI within the overweight but non-obese range is a surrogate for higher performance status or better overall health of the patient at time of cancer diagnosis, as described in prior studies [38]. Performance status itself has been associated with cancer survival in multiple disease sites [39]. Unfortunately, performance status was not able to be assessed directly in this retrospective study.

There are multiple advantages of this study including the large diverse nationwide sample size of PWLH with anal cancer seen in the equal access VA healthcare system, and the availability of the specific ART medications that the patients were taking during treatment. We employed a rigorous multi-data source design including utilizing data from an adjudicated national cancer registry database as well as confirmatory chart review performed by clinical oncologist. We also used a rigorous tiered approach for multivariable analyses to balance maximizing study power and reliability of effect 
estimates for PI use with observed outcomes while also adjusting for key or strong confounders. Further, our sensitivity analysis of excluded patients who had changed their ART medications within 90 days of starting radiation treatment found no significant difference in any survival or recurrence outcome in Cox regression analysis and thus supports the internal validity and robustness of study findings. However, this study has several limitations. The major limitation of this study is its retrospective nature, which precludes causal inferences and the inability to independently confirm adherence of patients taking their ART medications given our medical records-based study design. Additionally, given the relative rarity of anal cancer, while higher among PLWH, we had sample size-related power considerations that necessitated limiting the number of potential confounders we could account for in multivariable analyses. In addition, all PIs were grouped together in this analysis. It is possible that certain generations of the drug, or even specific PIs, have different individual effects. However, due to the paucity of research in this area and the limited sample size, it was decided that grouping all PIs together was the best initial approach. An inherent confounder in our analysis is the role of HIV in the natural course of anal carcinoma. HIV-infection has been shown to result in increased recurrence rates and overall worsened outcomes [3-5]. We attempted to control for HIV-related variables including era during which patients were treated as well as CD4 count. Interestingly, nadir CD4 count was the only HIV-related variable retained in any multivariable model, and was significantly associated with CFS. No HIV-related variable was found to be correlated with either OS or RFS. Larger multi-institutional studies are needed to confirm this given our limited sample size and retrospective nature. Nevertheless, as HIV-infected individuals are disproportionately affected by anal cell carcinoma and a large number are already taking PIs, these results remain applicable to this population.

This study constitutes one of the first analyses of PI use during CRT treatment for anal cell carcinoma. While in our report there was an increase in hospitalizations due to febrile neutropenia and other hematologic toxicities in patients taking PIs, their use was not associated with treatment variables associated with reduced survival, such as ability to complete the entire course of radiation or chemotherapy dose reduction. Importantly, the use of PIs was not associated with changes in outcomes. Our study suggests that PLWH and anal cancer on PIs who are receiving chemotherapy and radiation may benefit from receiving prophylactic granylocyte colony stimulating factor and close monitoring for hematologic toxicities to prevent hospitalizations. Further prospective and randomized clinical research needs to be done examining the association between specific PIs and their radiation sensitizing effects in both immunocompetent and HIV-infected populations. In addition, further optimization is needed in the treatment of anal cancer to improve overall outcomes as well as to reduce both acute and long-term toxicities.

\section{Conclusions}

The use of protease inhibitors during definitive CRT for the treatment of anal carcinoma in PLWH did not affect survival or recurrence outcomes. Their use was associated with increased hospitalizations due to hematologic toxicity, though not with any treatment variables associated with survival or recurrence. As HIV-infection continues to become a chronic disease, and the anal cancer disease burden increases, the treatment of anal cancer in PLWH will become more common. It is important for the medical community to understand the possible effects that drugs used to treat this disease, particularly potentially radiosensitizing drugs such as PIs, can have during radiation treatment.

\section{Abbreviations \\ PLWH: People living with HIV; ART: Antiretroviral therapy; CRT: Chemoradiation; NCCN: National Comprehensive Cancer Network; PI: Protease Inhibitor; VA: Department of Veterans Affairs; VINCI: VA Informatics and Computer Infrastructure; CDW: Corporate Data Warehouse; CCR: Central Cancer Registry; BMI: Body mass index; OS: Overall survival; RFS: Recurrence-free survival; CFS: Colostomy-free survival; PH: Proportional hazards; HR: Hazard ratio; Cl: Confidence interval; IQR: Interquartile range; IMRT: Intensity-modulated radiation therapy; adj: Adjusted; OR: Odds ratio}

\section{Acknowledgements}

Not applicable.

\section{Authors' contributions}

AY was a major contributor in writing the manuscript and performed the chart review. DW and DL were major contributors in writing the manuscript. YD performed the statistical analysis and helped to analyze the data. KR, JK, $\mathrm{PR}, \mathrm{CH}$ and SR extracted the data from the database and helped to analyze the data. $\mathrm{LL}$ and EC helped to analyze the data and were major contributors in writing the manuscript. All authors read and approved the final manuscript.

\section{Funding \\ This research work was supported by the National Cancer Institute (R01CA206476, PI: E. Chiao), the Houston Veterans Affairs Health Services Research and Development Center of Innovations (CIN13-413); and the Dan L. Duncan Cancer Center (P30CA022453). Dr. White received research support from the US Department of Veterans Affairs (CX001430, PI: D. White). The funders played no role is design, interpretation, or design to publish study findings.}

\section{Availability of data and materials}

We utilized the Corporate Data Warehouse (CDW Database) accessed through the VA Informatics and Computing Infrastructure (VINCI) available to researchers in the VA community (VINCI Database). Consistent with policies of the U.S. Department of Veterans Affairs, this is a closed databased that is not available to the public; therefore, the authors do not have the ability to provide the dataset. To conduct this study, we received administrative permission to access and use these data in the Ethics approval and consent to participate section of our IRB as granted by National Data Systems. 


\section{Declarations}

\section{Ethics approval and consent to participate}

This study was approved by the IRB at Baylor College of Medicine. Protocol Number $\mathrm{H}-38727$, Identifying novel pharmacologic risk factors for common non-AIDS defining cancers in individuals with well-controlled HIV infection.

\section{Consent for publication}

Not applicable.

\section{Competing interests}

The authors declare that they have no competing interests.

\section{Author details}

${ }^{1}$ University of Texas Health Science Center at Houston, McGovern School of Medicine, Houston, TX, USA. 'Department of Radiation Oncology, The University of Texas MD Anderson Cancer Center, Houston, TX, USA. ${ }^{3}$ Dartmouth College Geisel School of Medicine, Hanover, NH, USA. ${ }^{4}$ Department of Medicine, Baylor College of Medicine, 1155 Pressler St. Unit, Houston 1340, USA. ${ }^{5}$ Michael E. DeBakey Veterans Affairs Medical Center, Houston, TX, USA.

\section{Received: 14 September 2020 Accepted: 15 June 2021}

Published online: 05 July 2021

\section{References}

1. Siegel RL, Miller KD, Jemal A. Cancer statistics, 2019. CA Cancer J Clin. 2019; 69(1):7-34. https://doi.org/10.3322/caac.21551.

2. Nelson VM, Benson AB 3rd. Epidemiology of Anal Canal Cancer. Surg Oncol Clin N Am. 2017;26(1):9-15. https://doi.org/10.1016/j.soc.2016.07.001.

3. Palefsky JM, Holly EA, Efirdc JT, Da Costa M, Jay N, Berry JM, et al. Anal intraepithelial neoplasia in the highly active antiretroviral therapy era among HIV-positive men who have sex with men. Aids. 2005;19(13):140714. https://doi.org/10.1097/01.aids.0000181012.62385.4a.

4. Crum-Cianflone NF, Hullsiek KH, Marconi VC, Ganesan A, Weintrob A, Barthel RV, et al. Anal cancers among HIV-infected persons: HAART is not slowing rising incidence. 2010;24(4):535-43. https://doi.org/10.1097/QAD.0b013e32 $8331 \mathrm{f6e} 2$.

5. Pappou EP, Magruder JT, Fu T, Hicks CW, Herman JM, Fang S, et al. Prognostic and predictive Clinicopathologic factors of squamous Anal Cana Cancer in HIV-positive and HIV-negative patients: does HAART influence outcomes? World J Surg. 2018;42(3):876-83. https://doi.org/10.1007/s00268017-4201-6.

6. National Comprehensive Cancer Network. Anal Carcinoma (Version 1.2020). 2020. Available from: https://www.nccn.org/professionals/physician_gls/ pdf/anal.pdf. Accessed Mar 2020.

7. Kachnic LA, Winter K, Myerson RJ, Goodyear MD, Willins J, Esthappan J, et al. RTOG 0529: A Phase 2 Evaluation of Dose-Painted Intensity Modulated Radiation Therapy in Combination With 5-Fluorouracil and Mitomycin-C for the Reduction of Acute Morbidity in Carcinoma of the Anal Canal. Int J Radiat Oncol Biol Physics. 2013;86(1):27-33.

8. Ajani JA, Winter KA, Gunderson LL, Pedersen J, Benson AB 3rd, Thomas CR Jr, et al. Fluorouracil, mitomycin, and radiotherapy vs fluorouracil, cisplatin, and radiotherapy for carcinoma of the anal canal: a randomized controlled trial. Jama. 2008;299(16):1914-21. https://doi.org/10.1001/jama.299.16.1914.

9. James RD, Glynne-Jones R, Meadows HM, Cunningham D, Myint AS, Saunders MP, et al. Mitomycin or cisplatin chemoradiation with or without maintenance chemotherapy for treatment of squamous-cell carcinoma of the anus (ACT II): a randomised, phase 3 , open-label, $2 \times 2$ factorial trial. Lancet Oncol. 2013;14(6):516-24.

10. Goodman KA, Julie D, Cercek A, Cambridge L, Woo KM, Zhang Z, et al. Capecitabine With Mitomycin Reduces Acute Hematologic Toxicity and Treatment Delays in Patients Undergoing Definitive Chemoradiation Using Intensity Modulated Radiation Therapy for Anal Cancer. Int J Radiat Oncol Biol Physics. 2017;98(5):1087-95.

11. National Comprehensive Cancer Network. Anal Carcinoma (Version 2.2020). 2020. Available from: https://www.nccn.org/professionals/physician_gls/ pdf/anal.pdf. Accessed Nov 2020.

12. Ludmir EB, Kachnic LA, Czito BG. Evolution and Management of TreatmentRelated Toxicity in anal Cancer. Surg Oncol Clin N Am. 2017;26(1):91-113. https://doi.org/10.1016/j.soc.2016.07.004.
13. Gupta AK, Cerniglia GJ, Mick R, McKenna WG, Muschel RJ. HIV protease inhibitors block Akt signaling and Radiosensitize tumor cells Bothln vitroandln vivo. Cancer Res. 2005;65(18):8256-65. https://doi.org/10.1158/ 0008-5472.CAN-05-1220.

14. Gupta AK, Li B, Cerniglia GJ, Ahmed MS, Hahn SM, Maity A. The HIV protease inhibitor nelfinavir downregulates Akt phosphorylation by inhibiting proteasomal activity and inducing the unfolded protein response. Neoplasia. 2007;9(4):271-8.

15. Gupta AK, Lee JH, Wilke WW, Quon H, Smith G, Maity A, et al. Radiation response in two HPV-infected head-and-neck cancer cell lines in comparison to a non-HPV-infected cell line and relationship to signaling through AKT. Int J Radiat Oncol Biol Phys. 2009;74(3):928-33. https://doi. org/10.1016/j.jijobp.2009.03.004.

16. Pore N, Gupta AK, Cerniglia GJ, Jiang Z, Bernhard EJ, Evans SM, et al. Nelfinavir Down-regulates hypoxia-inducible factor $1 \mathrm{a}$ and VEGF expression and increases tumor oxygenation: implications for radiotherapy. Cancer Res. 2006;66(18):9252-9. https://doi.org/10.1158/0008-5472.CAN-06-1239.

17. Rengan R, Mick R, Pryma D, Rosen MA, Lin LL, Maity AM, et al. A Phase I Trial of the HIV protease inhibitor nelfinavir with concurrent chemoradiotherapy for unresectable stage IIIA/IIIB non-small cell lung cancer: a report of toxicities and clinical response. J Thorac Oncol. 2012;7(4): 709-15.

18. Rengan R, Mick R, Pryma DA, Lin LL, Christodouleas J, Plastaras JP, et al. Clinical outcomes of the HIV protease inhibitor nelfinavir with concurrent Chemoradiotherapy for Unresectable stage IIIA/IIIB non-small cell lung Cancer. JAMA Oncol. 2019;5(10):1464-72. https://doi.org/10.1001/jama oncol.2019.2095.

19. Alonso-Basanta M, Fang P, Maity A, Hahn SM, Lustig RA, Dorsey JF. A phase I study of nelfinavir concurrent with temozolomide and radiotherapy in patients with glioblastoma multiforme. J Neuro-Oncol. 2014;116(2):365-72. https://doi.org/10.1007/s11060-013-1303-3.

20. Garcia-Soto AE, McKenzie ND, Portelance L, Castillo RP, Pearson JM, Hu JJ, et al. Phase I trial of nelfinavir added to cisplatin chemotherapy with concurrent pelvic radiation for locally advanced cervical cancer. J Clin Oncol. 2015;33(15_suppl):TPS5619-TPS.

21. Garcia-Soto AE, McKenzie ND, Pearson JM, Portelance L, Lucci JA, Lin LL et al. Phase I trial of nelfinavir added to cisplatin chemotherapy with concurrent pelvic radiation for locally advanced cervical cancer. Gynecol Oncol. 2019;154:267-8. https://doi.org/10.1016/j.ygyno.2019.04.621.

22. Brunner TB, Geiger M, Grabenbauer GG, Lang-Welzenbach M, Mantoni TS, Cavallaro A, et al. Phase I trial of the human immunodeficiency virus protease inhibitor nelfinavir and Chemoradiation for locally advanced pancreatic Cancer. J Clin Oncol. 2008;26(16):2699-706. https://doi.org/10.12 00/JCO.2007.15.2355

23. Wilson JM, Fokas E, Dutton SJ, Patel N, Hawkins MA, Eccles C, et al. ARCII: a phase II trial of the HIV protease inhibitor nelfinavir in combination with chemoradiation for locally advanced inoperable pancreatic cancer. Radiother Oncol. 2016;119(2):306-11. https://doi.org/10.1016/j.radonc.2016. 03.021.

24. Hill EJ, Roberts C, Franklin JM, Enescu M, West N, Macgregor TP, et al. Clinical trial of Oral nelfinavir before and during radiation therapy for advanced rectal Cancer. Clin Cancer Res. 2016;22(8):1922-31. https://doi. org/10.1158/1078-0432.CCR-15-1489.

25. Curran WJ, Paulus R, Langer CJ, Komaki R, Lee JS, Hauser S, et al. Sequential vs concurrent Chemoradiation for stage III non-small cell lung Cancer: randomized phase III trial RTOG 9410. J National Cancer Institute. 2011; 103(19):1452-60. https://doi.org/10.1093/jnci/djr325.

26. Jackson GL, Melton LD, Abbott DH, Zullig LL, Ordin DL, Grambow SC, et al. Quality of nonmetastatic colorectal cancer care in the Department of Veterans Affairs. J Clin Oncol. 2010;28(19):3176-81. https://doi.org/10.1200/ JCO.2009.26.7948.

27. Zullig LL, Jackson GL, Dorn RA, Provenzale DT, McNeil R, Thomas CM, et al. Cancer incidence among patients of the U.S. veterans affairs health care system. Mil Med. 2012;177(6):693-701. https://doi.org/10.7205/MILMED-D-1100434.

28. Kramer JR, Hartman C, White DL, Royse K, Richardson P, Thrift AP, et al. Validation of HIV-infected cohort identification using automated clinical data in the Department of Veterans Affairs. HIV Med. 2019;20(8):567-70. https://doi.org/10.1111/hiv.12757

29. Gills JJ, Lopiccolo J, Tsurutani J, Shoemaker RH, Best CJM, Abu-Asab MS, et al. Nelfinavir, a lead HIV protease inhibitor, is a broad-spectrum, 
anticancer agent that induces endoplasmic reticulum stress, autophagy, and apoptosis in vitro and in vivo. Clin Cancer Res. 2007;13(17):5183-94.

30. Hampson L, Kitchener HC, Hampson IN. Specific HIV protease inhibitors inhibit the ability of HPV16 E6 to degrade p53 and selectively kill E6dependent cervical carcinoma cells in vitro. Antivir Ther. 2006;11(6):813-25.

31. Kim IA, Fernandes AT, Gupta AK, McKenna WG, Bernhard EJ. The influence of Ras pathway signaling on tumor radiosensitivity. Cancer Metastasis Rev. 2004;23(3-4):227-36. https://doi.org/10.1023/B:CANC.0000031763.95152.09.

32. Kim SH, Juhnn YS, Kang S, Park SW, Sung MW, Bang YJ, et al. Human papillomavirus 16 E5 up-regulates the expression of vascular endothelial growth factor through the activation of epidermal growth factor receptor, MEKV ERK1,2 and PI3K/Akt. Cell Mol Life Sci. 2006;63(7-8):930-8. https://doi. org/10.1007/s00018-005-5561-X.

33. See AP, Zeng J, Tran PT, Lim M. Acute toxicity of second generation HIV protease-inhibitors in combination with radiotherapy: a retrospective case series. Radiat Oncol. 2011;6:25.

34. Bower M, McCall-Peat N, Ryan N, Davies L, Young AM, Gupta S, et al. Protease inhibitors potentiate chemotherapy-induced neutropenia. Blood. 2004;104(9):2943-6. https://doi.org/10.1182/blood-2004-05-1747.

35. John M, Pajak T, Flam M, Hoffman J, Markoe A, Wolkov H, et al. Dose escalation in chemoradiation for anal cancer: preliminary results of RTOG 92-08. Cancer J Sci Am. 1996;2(4):205-11.

36. Konski A, Garcia M, John M, Krieg R, Pinover W, Myerson R, et al. Evaluation of Planned Treatment Breaks During Radiation Therapy for Anal Cancer: Update of RTOG 92-08. Int J Radiat Oncol Biol Physics. 2008;72(1):114-8.

37. Ben-Josef E, Moughan J, Ajani JA, Flam M, Gunderson L, Pollock J, et al. Impact of overall treatment time on survival and local control in patients with anal Cancer: a pooled data analysis of radiation therapy oncology group trials 87-04 and 98-11. J Clin Oncol. 2010;28(34):5061-6. https://doi. org/10.1200/JCO.2010.29.1351.

38. Dolan RD, Daly LE, Simmons CP, Ryan AM, Sim WM, Fallon M, et al. The relationship between ECOG-PS, mGPS, BMI/WL grade and body composition and physical function in patients with advanced Cancer. Cancers (Basel). 2020;12(5):1187. https://doi.org/10.3390/cancers12051187.

39. Jang RW, Caraiscos VB, Swami N, Banerjee S, Mak E, Kaya E, et al. Simple prognostic model for patients with advanced Cancer based on performance status. J Oncol Pract. 2014;10(5):e335-e41. https://doi.org/10.12 00/JOP.2014.001457.

\section{Publisher's Note}

Springer Nature remains neutral with regard to jurisdictional claims in published maps and institutional affiliations.

Ready to submit your research? Choose BMC and benefit from:

- fast, convenient online submission

- thorough peer review by experienced researchers in your field

- rapid publication on acceptance

- support for research data, including large and complex data types

- gold Open Access which fosters wider collaboration and increased citations

- maximum visibility for your research: over $100 \mathrm{M}$ website views per year

At $\mathrm{BMC}$, research is always in progress.

Learn more biomedcentral.com/submissions 